\title{
Conceptualization of Therapeutic Alliance During Psychiatric Residency Training
}

\author{
Stephen J. Cozza, M.D. \\ Walter Reed Army Medical Center, Washington DC
}

Follow this and additional works at: https://jdc.jefferson.edu/jeffjpsychiatry

Part of the Psychiatry Commons

Let us know how access to this document benefits you

\section{Recommended Citation}

Cozza, M.D., Stephen J. (1991) "Conceptualization of Therapeutic Alliance During Psychiatric Residency Training," Jefferson Journal of Psychiatry. Vol. 9 : Iss. 1 , Article 10.

DOI: https://doi.org/10.29046/JJP.009.1.008

Available at: https://jdc.jefferson.edu/jeffjpsychiatry/vol9/iss1/10

This Article is brought to you for free and open access by the Jefferson Digital Commons. The Jefferson Digital Commons is a service of Thomas Jefferson University's Center for Teaching and Learning (CTL). The Commons is a showcase for Jefferson books and journals, peer-reviewed scholarly publications, unique historical collections from the University archives, and teaching tools. The Jefferson Digital Commons allows researchers and interested readers anywhere in the world to learn about and keep up to date with Jefferson scholarship. This article has been accepted for inclusion in Jefferson Journal of Psychiatry by an authorized administrator of the Jefferson Digital Commons. For more information, please contact: JeffersonDigitalCommons@jefferson.edu. 


\title{
Conceptualization of Therapeutic Alliance During Psychiatric Residency Training
}

\author{
Stephen J. Cozza, MD
}

\begin{abstract}
This pilot study defines the Conceptualization of Therapeutic Alliance (CTA) as the psychotherapist's internalized construct of his ideal patient-therapist relationship based on the principles of Therapeutic Alliance, Working Alliance, and Helping Alliance. The study measures the CTA of third year medical students (MS3s), third year psychiatry residents (PGY3s), and consultant supervising psychotherapists (CSs) utilizing Fiedler's Ideal Therapeutic Relationship Scale. The CTA profiles of each experimental group are correlated with Fiedler's Ideal Therapeutic Relationship. Variance in CTA profiles within each group is also calculated. Preliminary results of this small study $(N=24)$ show that CTA measurements of the PGY3 group correlate more closely with Fiedler's Ideal Therapeutic Relationship and demonstrate less group variance than the CTA measurements of the MS3 group. This demonstrates that psychiatric residency training may encourage development of a cohesive CTA among residents of a given training class. No supervisory effect on the development of CTA among residents is distinguished.
\end{abstract}

\section{INTRODUGTION}

The term Therapeutic Alliance was introduced in a paper by Elizabeth Zetzel in 1956 (1). She described it as a recapitulation of the infant-mother relationship which allows the patient to give up inner defenses in the service of the analytic work. Rigidly interpreted, psychoanalytic theory might support the idea that this alliance is solely a manifestation of the patient's neurotic transference. However, Greenson described a similar phenomenon, the Working Alliance, as "the relatively nonneurotic, rational relationship between patient and analyst which makes it possible for the patient to work purposefully in the analytic situation" (2). He added that the analyst's style of working promotes the Working Alliance by producing an "analytic atmosphere" (2). In 1976 Luborsky coined the term Helping Alliance. He defined it as a "phenomenon reflecting the degree to which the patient experiences the relationship with the therapist as potentially helpful in achieving the patient's goals in psychotherapy" (3).

Location of Work and Reprints: Department of Psychiatry Room 2J49, Building 2 Walter Reed Army Medical Center Washington, D.C. 20307-5001

Acknowledgements: Special thanks to Dr. Morris Parloff for his guidance in preparation of this paper. The opinion or assertions contained herein are the private views of the author and are not to be construed as official or as reflecting the views of the Army or the Department of Defense. 
Throughout the psychoanalytic literature the development of a helpful therapeutic alliance between patient and therapist is valued as essential to effective treatment. Similarly, psychotherapy outcome studies support the relationship between the therapist's contribution to a good therapeutic alliance and positive treatment outcome $(3,4,5,6,7)$. Despite this evidence little is present in the literature that examines the effect of psychiatric residency training on the trainee's ability to promote good therapeutic alliances with patients.

In 1975 Fix and Haffke (8) examined psychiatric residents' abilities to do psychotherapy by measuring their level of functioning on Carkhuff and Berenson's "facilitative dimensions" of communication. This research showed no difference in level of communication with patients between residents at different levels of training. The instrument which was used, the Carkhuff Communication Scale, measures therapist involvement on different dimensions within the therapeutic relationship. In retrospect, the methodology of this study seems suboptimal. Residents were asked to participate in simulated "as if" therapy situations with previously videotaped patients. This may not have allowed true assessment of their skills, making the results of this study difficult to interpret.

Fix and Haffke's finding of a lack of measurable difference in psychotherapy skills could also indicate that differences in skill levels among psychiatric residents are small and escape measurement. Their assumption that measurable differences in psychotherapy skills should be present at different levels of residency training may not be true. It may be that the most significant changes which occur during residency training in regard to psychotherapy have to do with changes in how residents conceptualize therapeutic relationships. A change in skill level may lag behind this more fundamental ability.

There is considerable evidence to support this contention. Psychiatric residency training is a time of assimilating new concepts and developing new frameworks within which psychiatric patient care will be provided. Scanlon (9) discussed the crisis that psychiatric residents face in changing from a treatment system in which the caregiver does something "for" a patient to one in which he or she does something "with" a patient. By making such a distinction Scanlon defines one of the major tasks of psychiatric residency training as developing the ability to conceptualize the therapeutic alliance.

Similarly, in her classic book Learning Psychotherapy, Hilde Bruch describes psychotherapy "as a situation where two people interact and try to come to an understanding of one another with the specific goal of accomplishing something beneficial for the complaining person" (10). For Hilde Bruch learning psychotherapy is "the core, the very heart, of the psychiatric residency" (10). It is the process of learning to provide care by developing a method of interactive understanding. This appears to be another way of describing the process of integrating the concept of the therapeutic alliance.

Based on the principles of Therapeutic Alliance, Working Alliance and Helping Alliance, this study defines the Conceptualization of Therapeutic Alliance (CTA) as the psychotherapist's internalized construct of his idealized patient-therapist relation- 
ship. It is anticipated that psychiatric residency is a time in which significant development of CTA takes place.

It is not entirely clear how this occurs. The Group for the Advancement of Psychiatry (GAP) Report on Teaching Psychotherapy in Contemporary Psychiatric Residency Training (11) identifies supervision, didactic teaching, case conferences and group teaching as the methods by which psychotherapy is taught to psychiatric residents. Supervision is defined as a flexible process of routine meetings between student and supervisor. It is highlighted as a powerful traditional teaching method which addresses many clinical issues to include: (1) patient psychopathology and psychodynamics; (2) the importance of interpersonal sensitivity to the patient; (3) and the psychotherapeutic techniques suitable for the treatment of a particular patient.

In a survey of 400 residents in Canadian psychiatric residency training programs, a positive association was shown between the amount of supervision a resident is given and his evaluation of the training (12). Supervision was characterized as a parallel relationship which serves as a model to the resident-in-training for interpersonal functioning. The authors expected that the training therapist's involvement in a supervisory relationship founded on mutual respect would become a part of the trainee's working relationship with his patients. They hypothesized that as trainees spend more time with supervisors "they may be more likely to develop a better working alliance and feel more confident in their psychotherapeutic skills" (12).

The psychoanalytic literature similarly addresses the "parallelism" which exists between the supervisory relationship and treatment relationship. In a paper which examines the teaching of adolescent psychoanalytic psychotherapy to residents Sachs and Shapiro (13) identified the parallel emergence of adolescent issues in the supervisory setting. By thoroughly and empathically addressing these issues within the supervisory relationship, a framework was developed which modeled appropriate treatment for the adolescent patient within the treatment relationship.

Throughout this broad spectrum of psychiatric education literature supervision during psychiatric residency training is consistently highlighted as an important training tool. Supervised learning is not merely the simple didactic transfer of information from supervisor to resident. It is the interpersonal nature of the supervisor-trainee relationship that is emphasized as imperative to the development of CTA in psychiatry residents.

In his work examining models of therapeutic relationships Fiedler developed a scale for profiling therapist conceptualization of therapeutic alliance (14). He asked therapists to describe what they considered to be their ideal therapeutic relationships. This seems in accordance with the definition of CTA described earlier. Fiedler found that the "ideal" profiles of expert therapists of different schools of psychotherapy were more similar to each other than they were to the ideals of less experienced therapists from their own schools (14). In his paper he included this profile and defined it as the Ideal Therapeutic Relationship. He maintained that this is the profile conceptualized by all therapists as expertise increases. Fiedler's work provides an instrument which allows profiling of a therapist's CTA, as well as furnishes a 
singular expert CTA, the Ideal Therapeutic Relationship, with which it can be compared. In light of Fiedler's work, CTA development during psychiatric residency training could be viewed as the progressive approach a therapist makes toward this distinctive CTA of greater expertise. Supervision would be expected to impact positively in this regard.

\section{HYPOTHESES}

In the current investigation the following hypotheses are considered:

1. As psychiatric residency training is believed to assist in the development of CTA, then the measured CTA profiles of third year psychiatric residents-intraining (PGY3s) will more closely approximate Fiedler's Ideal Therapeutic Relationship than those of third year medical students (MS3s).

2. Since supervisors are considered important contributors to resident development of CTA, then it is expected that CSs will more closely approximate Fiedler's Ideal Therapeutic Relationship than will PGY3s.

3. Since it is expected, in agreement with Fiedler, that increasing expertise results in a more uniform CTA, then the variance of CTA profiles within each group will decrease with increasing expertise (MS3 > PGY3 > CS).

\section{METHODOLOGY}

Instrument: Fiedler's Ideal Therapeutic Relationship Scale (14) was selected as the instrument for measurement of CTA. Fiedler's scale consists of 75 single statements printed on separate cards. Each card describes a therapist's involvement in a relationship with a patient. The cards comment on the patient-therapist relationship along three dimensions and twenty five different cards address each of these three axes: (1) communication between therapist and patient; (2) emotional distance between therapist and patient; and (3) therapist/patient status within the relationship. Cards contain statements which are therapeutic, neutral, and countertherapeutic. Example statements include: “The therapist's remarks fit in just right with the patient's mood and content"; "The therapist is pleasant to the patient"; "The therapist always apologizes when making a remark"; and "The therapist talks down to the patient as if he were a child."

The cards are sorted by each research subject from most to least characteristic of his ideal therapeutic relationship. Cards are sorted and scored according to Stephenson's Q-technique (15). By using this technique of Q-sorting a distribution is developed in which 1 card is selected as most characteristic, 7 cards selected as very characteristic, 18 as somewhat characteristic, 23 are selected in a middle or neutral category, 18 as somewhat inapplicable, 7 as uncharacteristic and 1 card as least characteristic of an ideal therapeutic relationship. Although some statements are clearly positive and others clearly negative, this procedure requires the subject to go beyond simply assigning valence to a statement. It requires that a subject compare 
cards of similar valence and rank them amongst themselves. By sorting the cards in such a way each subject generates a profile of his CTA. This can then be correlated with Fiedler's Ideal Therapeutic Relationship (the specific standardized sorting which experts in Fiedler's study defined) or it can be compared to another subject's profile.

Subjects: Eight third year medical students (MS3s) randomly selected from a group of students during their psychiatry clerkships, the complete class of eight psychiatry residents completing their third year of training (PGY3s), and eight consultant supervisors (CSs) at a tertiary care medical center were asked to complete the Fiedler Ideal Therapeutic Relationship Scale. The CS group consisted of 7 MDs and $1 \mathrm{PhD}$ who have been practicing psychotherapists for more than 15 years. These CSs also serve as psychotherapy supervisors to the PGY3 group. MS3s and PGY3s were selected as pre- and post-training representatives.

Data Collection: Each participant was instructed by the author (sjc) on how to use the instrument and was asked to describe his "hypothetical, ideal therapeutic relationship" by sorting Fiedler's 75 cards as described previously. This profile is considered a description of the individual's CTA. Each subject's CTA was correlated with Fiedler's Ideal Therapeutic Relationship. The three subject group (MS3, PGY3, and C.S) correlation results were compared with each other using the Student T-test analysis after Fisher's z-transformation. CTA profile variance was calculated within each group. Variances between groups (MS3-PGY3; MS3-CS; PGY3-CS) were compared using Bartlett's Test of Homogeneity of Variance.

\section{RESULTS}

Table 1 lists correlation coefficients and Fisher's z-transformations of group subjects' CTAs with Fiedler's Ideal Therapeutic Relationship. Means of z-trans-

TABLE 1.

Correlation Coefficients of Subject Results (r) With Fiedler's Ideal and Fisher z-Transformations*

\begin{tabular}{ccrcrcr}
\hline Subj \# & \multicolumn{2}{c}{ MS3 } & \multicolumn{2}{c}{ PGY3 } & \multicolumn{2}{c}{ CS } \\
& $\mathrm{r}$ & $\mathrm{z}(\mathrm{r})$ & $\mathrm{r}$ & $\mathrm{z}(\mathrm{r})$ & $\mathrm{r}$ & $\mathrm{z}(\mathrm{r})$ \\
\hline 1 & .78 & 1.045 & .85 & 1.256 & .83 & 1.188 \\
2 & .75 & .973 & .83 & 1.188 & .82 & 1.157 \\
3 & .70 & .867 & .82 & 1.157 & .79 & 1.071 \\
4 & .70 & .867 & .82 & 1.157 & .75 & .973 \\
5 & .68 & .829 & .77 & 1.020 & .70 & .867 \\
6 & .66 & .793 & .75 & .973 & .67 & .811 \\
7 & .63 & .741 & .73 & .929 & .67 & .811 \\
8 & .51 & .563 & .70 & .867 & .49 & .536 \\
Mean & .68 & .835 & .78 & 1.068 & .72 & .927 \\
Stan Dev & & .146 & & .140 & & .216 \\
\hline
\end{tabular}

$*_{z}=1 / 2 \ln ((1+r) /(1-r))$ 
formed correlation coefficients for the MS3, PGY3, and CS groups are .835, 1.068, and .927 respectively. Standard deviations are $.146, .140$, and .216 for these respective groups.

Table 2 shows calculated Student-T values when group z-transformed correlation coefficients were compared indicating that CTAs of PGY3s correlate at a significantly higher level with Fiedler's Ideal $(p<.005)$ than CTAs of MS3s. No significant difference in correlation was noted between CSs and PGY3s or between CSs and MS3s.

Table 3 lists intragroup response variances for MS3s, PGY3s, and CSs (.514, .291 , and .380 respectively). Variances between groups (MS3-PGY3; MS3-CS; PGY3CS) were compared using Bartlett's Test of Homogeneity of Variance. Calculated values for Bartlett's Test of Homogeneity of Variance are listed in Table 3. Chisquare values were calculated and are listed in Table 3 as well $(a=2 ; f=600$, degrees of freedom $=1$ ). Significant differences in intragroup response variance were noted at the $\mathrm{p}<.005$ level for each compared group dyad. The MS3 group showed significantly greater variance of CTA profiles than did the CS group. The CSs showed significantly more variance than did PGY3s.

\section{DISCUSSION}

The results of this investigation should be interpreted cognizant of the small sample size. It is also important to consider the possible limitations of the instrument used. More recent instruments are available to measure therapist contribution to the therapeutic alliance $(3,6)$. However, these instruments measure therapist skill in relation to treatment outcome, and do not lend themselves to measurement of conceptualization as does Fiedler's instrument. Fiedler's scale is well-suited to this investigation. His initial paper discussing the instrument (14) was followed by two additional publications using the same instrument $(16,17)$. In the first of these later papers (16) Fiedler demonstrated that the actual observed therapeutic relationships created by experts more closely approximated the Ideal Therapeutic Relationship that those created by non-experts. In the last paper (17) four multiple factor analyses of the data from the previous study were described. Although no large studies have been completed which address the reliability and reproducibility of these results, Fiedler's findings were consistent within these three publications.

TABLE 2.

Calculated Student-t Values and p Values of Inter-Group Result Comparisons*

\begin{tabular}{lcc}
\hline Subject Groups & $\mathrm{t}$ & $\mathrm{p}$ \\
\hline MS3 - PGY3 & 3.26 & $\mathrm{p}<.005^{* *}$ \\
MS3 - CS & 1.00 & $\mathrm{p}>.1$ \\
PGY3 - CS & 1.55 & $\mathrm{p}>.1$ \\
\hline
\end{tabular}

*Using 7 degrees of freedom

**Significant difference noted 
TABLE 3.

Intra-Group Variance and Bartlett's Test of Homogeneity of Variance

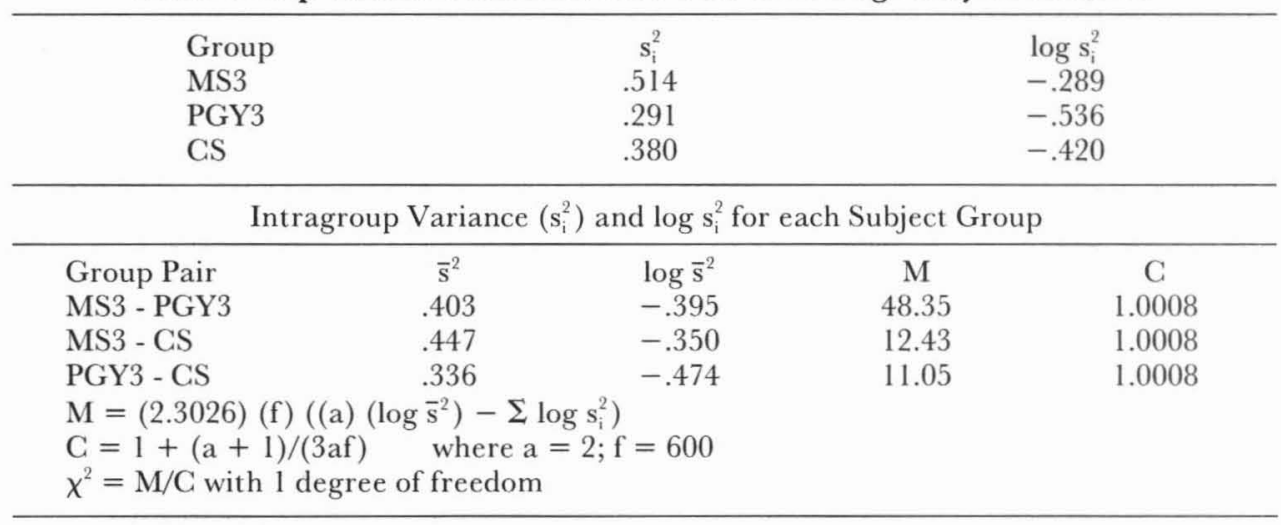

Calculated values for Bartlett's Test of Homogeneity of Variance

\begin{tabular}{lcc}
\hline Group Pair & $\chi^{2}$ & $\mathrm{p}$ \\
MS3 - PGY3 & 48.31 & $\mathrm{p}<.005^{* *}$ \\
MS3 - CS & 12.42 & $\mathrm{p}<.005^{* *}$ \\
PGY3 - CS & 11.04 & $\mathrm{p}<.005^{* *}$ \\
\hline
\end{tabular}

Chi-Square values $\left(\chi^{2}\right)$ for Bartlett's Test of Homogeneity of Variance and $p$ values

**shows significant difference

As was anticipated in Hypothesis 1, CTA profiles of PGY3s more closely approximate Fiedler's Ideal Therapeutic Relationship than those of MS3s. The data suggest that the CTAs of PGY3s are more similar to experts than those of MS3s. It is assumed that any effect which occurs in the time between MS3 and PGY3 years can be attributed to psychiatric residency training. The results advance the hypothesis that psychiatric residency training through the PGY3 year contributes significantly to CTA development.

It might be argued that the psychiatric specialty preselects those medical students who more closely correlate with Fiedler's Ideal prior to training. This plausible explanation was not in fact supported by the data of this study. Those subjects in the MS3 group whose CTA profiles correlated at higher levels with Fiedler's Ideal (.75 and .78) had made decisions not to specialize in psychiatry. In fact, it was the student whose CTA profile correlated least (.51) who was most seriously considering psychiatry as a specialty.

There was no significant difference in the degree to which the CTA profiles of PGY3s and CSs correlated with Fiedler's Ideal. This is not supportive of Hypothesis 2. It might be argued that such a difference does not exist because the process of supervision results in complete equilibration of expertise between PGY3s and CSs. 
This seems highly unlikely. Furthermore, a lack of a significant difference between CS and MS3 degrees of CTA correlation with Fiedler would then need to be interpreted that no difference in expertise exists between MS3s and CSs as well. Assuming that the CS group is that group with the most psychotherapeutic expertise, and that CS CTA profiles would reflect this, then it would appear that Fiedler's instrument was unable to measure this difference.

Some observations during this investigation indicate that this might be true. Of the three groups tested, the CSs were most verbal about their difficulty in describing their CTA profiles with Fiedler's cards. Fiedler's Ideal Relationship Scale contains statements which were described as "general" and "ambiguous." This complaint was most often made by CSs. It is this investigator's conclusion that a highly trained therapist develops a very subtle communication system in working with patients. It appeared that each card had a greater breath of interpretation for CSs as compared to PGY3s or MS3s. Fiedler's instrument did not appear to provide the complex framework needed to accurately measure the CTA profiles of the CS group.

It is difficult to determine whether supervision has much of an effect on the development of CTA among psychiatric residents based on the results of this investigation. Whatever supervisory effect does exist may be difficult to ascertain using Fiedler's instrument; it is possible that at higher levels of expertise the reliability of the scale breaks down. Although the findings suggest that residents do not simply internalize the CTA profiles of their supervisors, as the measured CTA profiles of both groups were markedly dissimilar, the small sample size is a limitation with regard to drawing more definitive conclusions.

Intragroup CTA profile variance was significantly lower for the PGY3 group than the MS3 group, supporting Hypothesis 3. This supports the inference, independent of Fiedler's Ideal, that PGY3s agreed more among themselves than did MS3s about how therapist-patient relationships are conceptualized. The development of a more uniform expert group of CTA profiles among PGY3s than MS3s would support residency training as a possible causative agent.

More unexpectedly, intragroup CTA profile variance is significantly higher in the CS group than the PGY3 group. This is in clear contrast to Fiedler's original finding and contradicts Hypothesis 3. This may be a result of the previously mentioned difficulty with Fiedler's instrument accurately measuring CS CTA profiles. The explanation also may be that experts conceptualize therapeutic alliances in a variety of ways. Eaton critiqued Fiedler's and others' work in defining ideal therapeutic relationship characteristics (18). He questioned the existence of such a singular "ideal." He commented anecdotally that the experienced therapist understands the need for flexibility within the treatment relationship and that no single mode of interacting would suffice for all patients:

Models of the appropriate therapeutic relationship can, therefore, involve a great variety of roles and communication processes. Influence may be brought to bear by being nondirective, interpretive, authoritative, non- 
judgemental, reassuring, moralizing, or some combination of these and other role interactions (18).

The significantly lower variance measured in the PGY3 group compared to the MS3 group seems to support Fiedler's assertion that increasing expertise results in a more uniform CTA profile. However, this is not borne out when comparing the CTA profiles of the PGY3 and CS groups. The soundness of Eaton's criticism may be reflected in the higher CTA profile variance of the CS group.

The causes of such homogeneity of response within the PGY3 group may be multiple. Again, selection of applicants for a specific program might account for this phenomenon. But, it also seems likely that such a finding results from uniform training experiences. Similar didactic training, case conferences, patient population, faculty members, and supervisors all would serve to make the training experience similar within a program.

As mentioned in the introduction, interpersonal interaction, particularly in supervision, is acknowledged as the forum in which development of CTA is believed to largely occur. However, the higher CTA profile variance in the CS group makes the effect of supervision an unlikely contributor to the low CTA profile variance observed in the PGY3 group. Other important, interpersonal interactions in a residency class are the relationships between the trainees themselves. A class of residents may develop CTA by consistently processing ongoing didactic, clinical and supervisory material in their day to day interactions. This core CTA, developed at the resident level, may serve as a nidus for the future assimilation of more intricate interpersonal relationship principles.

A model for the progressive phases of CTA development might include the following: 1) entry of untrained therapists into a psychiatric residency training program with varied interpersonal experiences and pretraining CTAs (as exemplified by MS3s in this study), 2) the development of a cohesive CTA within the interpersonal sphere of the residency class as it progresses through training, and 3) the post-training modification of this CTA through individual experience which, in the therapist of higher expertise, results in the development of a flexible, sophisticated CTA that becomes complicated to measure.

\section{FUTURE RESEARCH}

This pilot study measured differences in Conceptualization of Therapeutic Alliance (CTA) within one residency training program at three cross-sections of training. Many variables are present that make it necessary to be cautious in drawing firm conclusions. Primarily, this study underlines the need for future research in this area involving larger numbers of subjects.

The finding of a cohesive CTA among the PGY3s in this study raises the question as to whether this phenomenon is unique to either the subject group or the subject training program. Comparison of different training programs would provide helpful information. Should such cohesive CTAs be identified within other programs it would be interesting to see if they differ from program to program. 
Future research needs to address whether the development of such a cohesive CTA is a part of the natural stage of development of an expert therapist, as suggested in this study. In other training programs it may not be a natural part of therapist development. Benefits and limitations of different therapist developmental pathways could be considered, and training could be altered to optimize therapist development.

Future longitudinal studies should include measurement of individual therapist CTA at different times before and during training. Such an approach would reduce the number of confounding variables and provide greater information on how training effects an individual's CTA over time. A control group might include residents in other medical specialties to determine if changes in CTA occur during psychiatric residency training or residency training in general.

Lastly, the difficulty in measurement of a therapist's CTA needs to be examined. Glearly, Fiedler's Ideal Therapeutic Relationship Scale needs more rigorous testing of reliability and validity. In addition, other techniques need to be constructed that allow inspection of the complex and intricate interpersonal relationship systems upon which expert therapists appear to rely. This would not only allow for measurement and evaluation of experienced therapists' CTAs, but could also serve as a tool to examine the interpersonal teaching effect of the resident-supervisor relationship.

\section{SUMMARY}

In summary, the findings of this research support the hypothesis that psychiatric residency training affects the development of Conceptualization of Therapeutic Alliance (CTA). Psychiatric residents (PGY3s) were shown to correlate with Fiedler's Ideal Therapeutic Relationship to a greater degree than untrained medical students (MS3s). Cohesiveness of response within the PGY3 group, in comparison to MS3s and CSs, suggests a cohort effect in which a residency training class develops a unified CTA. It seems that Fiedler's Ideal Relationship Scale becomes less capable of measuring CTA as expertise increases. An effect of supervision on the development of CTA in residents was not measured. A model for the progressive phases of CTA development is suggested. Future research is also considered.

\section{REFERENCES}

1. Zetzel ER: Current concepts of transference. Int J Psychoanalysis 37:369-376, 1956

2. Greenson RR: The Technique and Practice of Psychoanalysis, Vol. 1. Madison, International Universities Press, 1967

3. Luborsky $\mathrm{L}$ et al: Two helping alliance methods for predicting outcomes of psychotherapy, A counting signs vs. a global rating method. J Nerv Ment Dis 171:480-91, 1983

4. Foreman SA, Marmar CR: Therapist actions that address initially poor therapeutic alliances in psychotherapy. Am J Psychiatry 142:922-6, 1985

5. Luborsky L et al: Therapist success and its determinants. Arch Gen Psych 42:602-11, 1985

6. Marziali EA: Prediction of outcome of brief psychotherapy from therapist interpretive interventions. Arch Gen Psych 41:301-4, 1984 
7. Marziali EA: Three viewpoints on the therapeutic alliance, similarities, differences, and associations with psychotherapy outcome. J Nerv Ment Dis 172:417-23, 1984

8. Fix AJ, Haffke EA: Relationship between psychotherapy skills and level of training in a psychiatric residency program. Soc Sci Med 489-91, 1975

9. Scanlon JM: Physician to student: The crisis of psychiatric residency training. Am J Psychiatry 128:1107-11, 1972

10. Bruch H: Learning psychotherapy rationale and ground rules. Cambridge, Mass, Harvard University Press, 1974

11. Group for the Advancement of Psychiatry, Committee on Therapy: Teaching psychotherapy in contemporary psychiatric residency training. Rep/Grp Adv Psychiatry 120:1-56, 1987

12. Perez EL, Krul LE, and Kapoor R: The teaching of psychotherapy in Canadian psychiatric residency programs: Resident's perceptions. Can J Psychiatry 29:658-64, 1984

13. Sachs DM, Shapiro SH: Comments on teaching psychoanalytic psychotherapy in a residency training program. Psychoanal Quar 43:51-76, 1974

14. Fiedler FE: The concept of an ideal therapeutic relationship. J Consult Psychol 14:239-45, 1950

15. Stephenson W: The study of behavior Q-technique and its methodology. Chicago, Ill., The University of Chicago Press, 1953

16. Fiedler FE: A comparison of therapeutic relationships in psychoanalytic, nondirective and Adlerian therapy. J Consult Psychol 14:436-445, 1950

17. Fiedler FE: Factor analyses of psychoanalytic, nondirective, and Adlerian therapeutic relationships. J Consult Psychol 15:32-38, 1951

18. Eaton JW: The client-practitioner relationship as a variable in the evaluation of treatment outcome. Psychiatry 22:189-95, 1959 